\title{
EUROfusion
}

EUROFUSION WPMST1-CP(16) 15539

CJ Rapson et al.

\section{Experiments on Actuator Management and Integrated Control at ASDEX Upgrade}

Preprint of Paper to be submitted for publication in Proceedings of 29th Symposium on Fusion Technology (SOFT

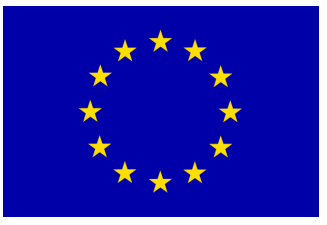

This work has been carried out within the framework of the EUROfusion Consortium and has received funding from the Euratom research and training programme 2014-2018 under grant agreement No 633053. The views and opinions expressed herein do not necessarily reflect those of the European Commission. 
This document is intended for publication in the open literature. It is made available on the clear understanding that it may not be further circulated and extracts or references may not be published prior to publication of the original when applicable, or without the consent of the Publications Officer, EUROfusion Programme Management Unit, Culham Science Centre, Abingdon, Oxon, OX14 3DB, UK or e-mail Publications.Officer@euro-fusion.org

Enquiries about Copyright and reproduction should be addressed to the Publications Officer, EUROfusion Programme Management Unit, Culham Science Centre, Abingdon, Oxon, OX14 3DB, UK or e-mail Publications.Officer@euro-fusion.org

The contents of this preprint and all other EUROfusion Preprints, Reports and Conference Papers are available to view online free at http://www.euro-fusionscipub.org. This site has full search facilities and e-mail alert options. In the JET specific papers the diagrams contained within the PDFs on this site are hyperlinked 


\title{
Experiments on Actuator Management and Integrated Control at ASDEX Upgrade
}

C.J. Rapson ${ }^{\mathrm{a}}$, F. Felicib ${ }^{\mathrm{b}}$, C. Galpertic, P.T. Lang ${ }^{\mathrm{a}}$, M. Lennholmd, E. Maljaars ${ }^{\mathrm{b}}$, M. Maraschek ${ }^{\mathrm{a}}$, B. Plöckl ${ }^{\mathrm{a}}$, M. Reich $^{\mathrm{a}}$, O. Sauter ${ }^{\mathrm{c}}$, J. Stober ${ }^{\mathrm{a}}$, W. Treutterer ${ }^{\mathrm{a}}$, the ASDEX Upgrade team ${ }^{\mathrm{a}}$, and the EUROfusion MST1 team ${ }^{\mathrm{e}}$

\author{
${ }^{a}$ Max Planck Institute for Plasma Physics, Boltzmannstrasse 2, 85748 Garching, Germany \\ ${ }^{b}$ Eindhoven University of Technology, P.O. Box 513, 5600 MB Eindhoven, The Netherlands \\ ${ }^{c}$ École Fédérale Polytechnique de Lausanne (EPFL), Swiss Plasma Center (SPC), Lausanne, Switzerland \\ ${ }^{d}$ CCFE, Culham Science Centre, Abingdon, OX14 3DB, UK \\ ${ }^{e}$ See http://www.euro-fusionscipub.org/mst1.
}

\begin{abstract}
The established field of integrated multivariable control promises improved performance and stability for strongly coupled processes, given a control-oriented model of the system. Fusion plasmas are strongly coupled, but there is currently no model which accurately reflects the nature of these complex interactions. Therefore, experiments were performed specifically to investigate coupling between controlled parameters, as a step towards designing integrated controllers in the future. The parameters chosen were core density, divertor neutral pressure and divertor temperature. For control of the plasma pressure and Neoclassical Tearing Modes, where a simple model of the coupling is known, it will be shown that linking the two controllers gives reliably good plasma performance.

An additional complication with integrated control is that limited actuator resources are often oversubscribed when trying to control multiple parameters simultaneously. In order to achieve the optimum result, some form of actuator management is a pre-requisite for integrated control. An algorithm has been developed to automatically allocate Electron Cyclotron Resonant Heating gyrotrons to targets, by evaluating a cost function in real-time. Results will be shown to demonstrate the flexibility of this routine to changes in plasma state, gyrotron availability and the goals of the physics experiment.
\end{abstract}

Keywords: actuator management, integrated control, ECRH, NTM, density, pellet, radiation, DCS, ASDEX Upgrade

\section{Introduction}

Feedback control is generally employed to improve the performance and reliability of a system. For coupled systems, integrated control of multiple parameters can improve control or in some cases may even be necessary for stability. Aeroplanes are an intuitive example, where changes in roll, pitch or yaw are controlled by moving various flaps. The changing aerodynamics affects not only the direction of flight but also the speed, which must be compensated by adjusting the thrust actuator. Industrial processes [e.g 1, 2], robotics [e.g. 3] and many other fields have also profited from multivariable control.

Attempts have been made to apply integrated control to fusion experiments [e.g. 4], however the lack of control-oriented models which are valid across a range

Email address: chris.rapson@ipp.mpg.de (C.J. Rapson) of scenarios is a challenging handicap. Even the most complex models struggle to capture coupling between multiple parameters and chaotic bifurcations between plasma states (e.g. L-/H-mode, type I/II/III ELMs, sawtooth/fishbone/no central MHD, ITG/TEM turbulence, impurity accumulation/expulsion, attached/detached divertor etc.) This paper describes a series of experiments using up to 5 SISO (single input, single output) feedback controllers. Observations on the couplings between these controllers will be presented here, and used to develop further integrated controllers in the future. For two of these controllers - plasma pressure $(\beta)$ and Neoclassical Tearing Mode (NTM) - a simple model can describe the coupling and the two controllers can be coordinated by having them react to the same event.

An additional challenge for integrated control is that actuators are not only coupled, but scarce. In order to achieve control objectives as closely as possible within the available resources, some form of actuator manage- 
ment is needed. The design for an algorithm for actuator management of Electron Cyclotron Resonant Heating (ECRH) [5] has now been successfully deployed in experiments, and these results will be presented here.

\section{Actuator Management}

\subsection{Algorithm Design Recap}

ECRH is a good candidate for actuator management routines because it is a flexible actuator, capable of addressing several targets. It can be used to control the temperature and current profile, as well as sawteeth and NTMs. In metal-walled machines, central ECRH (or Ion Cyclotron Resonant Heating) is critical to avoid tungsten accumulation. The priority for the various targets can change during a discharge, as can the availability of the gyrotrons if they trip. An algorithm has been developed which uses a cost function to determine the optimal allocation of gyrotrons to targets at each time step. Based on this allocation, pairs of low level controllers control the launcher mirrors and power for each gyrotron. The cost function penalises launcher movement (to always use the closest gyrotron), switching (to discourage bouncing between two similar solutions) and the number of active gyrotrons. The cost function rewards power supplied which approaches or exceeds the required power $\left(P_{\text {reqd }}\right)$ and uses a multiplier which indicates the relative importance of each target (imp). The list of targets, their imp and $P_{\text {reqd }}$ are configuration settings which can be changed from shot to shot to reflect the goals of the physics experiment. You can find out more about the actuator management scheme in ref. [5].

\subsection{Results}

By changing configuration settings over 20 shots in the recent ASDEX Upgrade campaign, the algorithm demonstrated its reliability and a range of useful functions. A representative overview is given by the comparison of \#33094 and \#33101 in fig. 1. By changing $P_{\text {reqd }}$ for central heating from $1 \mathrm{MW}$ to $400 \mathrm{~kW}, 1 \mathrm{gy}-$ rotron rather than 2 were allocated to this target. The initial positions of the gyrotrons are reversed, and in both cases the nearest gyrotrons were chosen.

Two of the targets are for pre-emptive control of NTMs. In \#33094 the settings were imp $=\{15,20\}$ for pre-empting 3/2 and 2/1 NTMs respectively. Therefore one gyrotron tracks each of the $\mathrm{q}=1.5$ and $\mathrm{q}=2.0$ surfaces and power is applied. In \#33101 imp $=\{0,0\}$ such that the outer gyrotrons are idle until an NTM is detected at $\mathrm{t}=2.6 \mathrm{~s}$. The appearance of a $2 / 1 \mathrm{NTM}$ is a

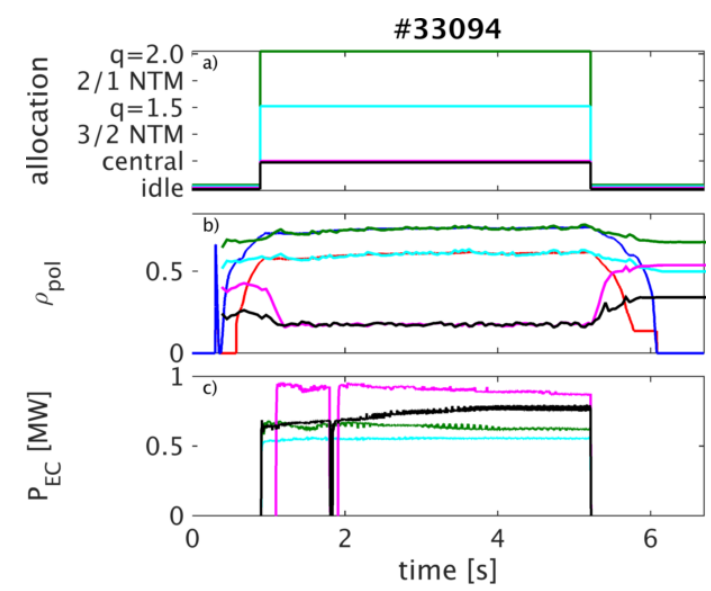

\#33101

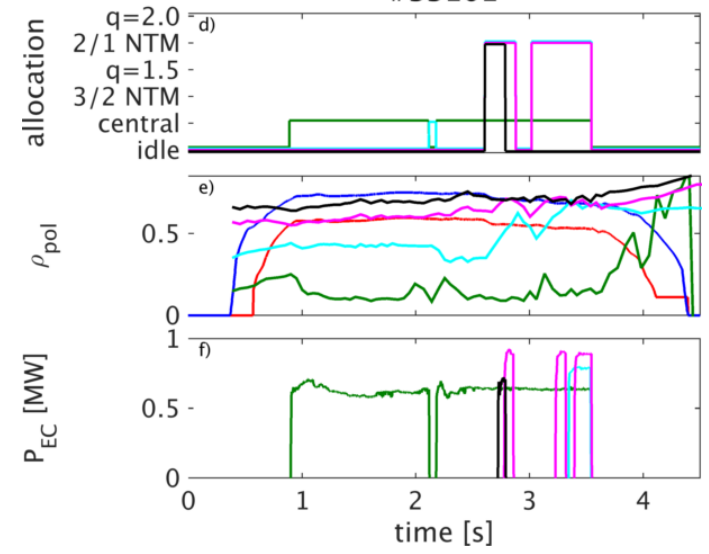

Figure 1: Comparison of ECRH actuator allocation for two AUG shots. In each case the top frames $(\mathrm{a}, \mathrm{d})$ show to which target each gyrotron is allocated. Each of the 4 gyrotrons is plotted with a different colour (black, magenta, cyan, green). The next frames (b,e) show the deposition location of the gyrotrons, along with the position of the $\mathrm{q}=1.5$ and $\mathrm{q}=2.0$ surfaces. The bottom frames $(\mathrm{c}, \mathrm{f})$ show the power supplied by each of the gyrotrons.

dramatic change of the plasma state, and the algorithm responds by allocating 3 gyrotrons to this target.

Finally, the algorithm demonstrated its response to tripping gyrotrons. In \#33101 the central (green) gyrotron trips at $\mathrm{t}=2.1 \mathrm{~s}$ and the closest idle gyrotron (cyan) is selected to replace it. The cyan line starts moving towards the centre while the green line returns to its start position. However, before they can cross, the gyrotron is successfully re-activated and the original allocation is resumed. When the black gyrotron trips at $\mathrm{t}=2.8 \mathrm{~s}$ there is no suitable replacement, likewise for the black and magenta gyrotrons at $\mathrm{t}=1.8 \mathrm{~s}$ in \#33094.

\subsection{Future Work}

The algorithm is being ported to TCV. Due to the physics base, configurability and the use of Simulink 
for rapid prototyping, it is hoped that this algorithm can demonstrate its flexibility to work on different ECRH setups. In future, it is envisaged to make $P_{\text {reqd }}$ and $\mathrm{imp}$ time varying signals. For example, the $P_{\text {reqd }}$ for central heating could be inversely proportional to the density and the importance of NTM control could increase with $\beta$. One could also combine pre-emption and stabilisation of an NTM into a single target, with the importance increasing once an NTM is detected. Since the number of combinations scales as $\left(n_{\text {targets }}\right)^{n_{g y r}}, 2$ fewer targets would reduce the number of combinations from 1296 to 256. The extra capacity could be used for new targets such as sawtooth control. Even with these improvements, the scaling to 24 gyrotrons foreseen for ITER will require new strategies, some of which are being investigated as part of a PhD Thesis [6].

\section{Working Towards Integrated Control}

\subsection{Linking $\beta$ and NTM Control}

The coupling of NTMs and $\beta$ can be described by a simple model. NTMs are pressure driven, hence increasing $\beta$ will increase both the likelihood of triggering an NTM, and the size of an existing one. NTMs increase transport, so drive down $\beta$ in proportion to their size. Most high $\beta$ scenarios developed in feedforward have been limited or even disrupted by NTMs. Therefore the references to the two controllers were linked, such that if no NTM is detected, the $\beta$ reference ramps up while the NTM controller can either perform preemptive control or be idle. When an NTM is detected, the $\beta$ reference ramps down and NTM stabilisation is activated. The effectiveness of this strategy can be seen in fig. 2 where the stabilisation of the NTM by ECRH is aided by the reduction in $\beta$ from $\mathrm{t}=1.8-2.4 \mathrm{~s}$. Afterwards, normalised $\beta_{N}$ is increased to 2.6 before the discharge is brought to a planned end. It was later thought that it may be even more optimal to maintain a constant heating power during the NTM, to avoid such a large drop in $\beta$ and to have more stable plasma conditions. The result shown in fig. 3, which will be discussed in more detail in section 3.2, seems to confirm this hypothesis.

\subsection{Observation of Couplings}

In addition to the $\beta$ and NTM controllers, 3 more SISO controllers were operated simultaneously to investigate any coupling. The parameters controlled were core density using $D_{2}$ pellets [7], divertor neutral pressure $n 0$ div using $D_{2}$ gas and divertor temperature $T_{\text {div }}$ using $N_{2}$ gas.
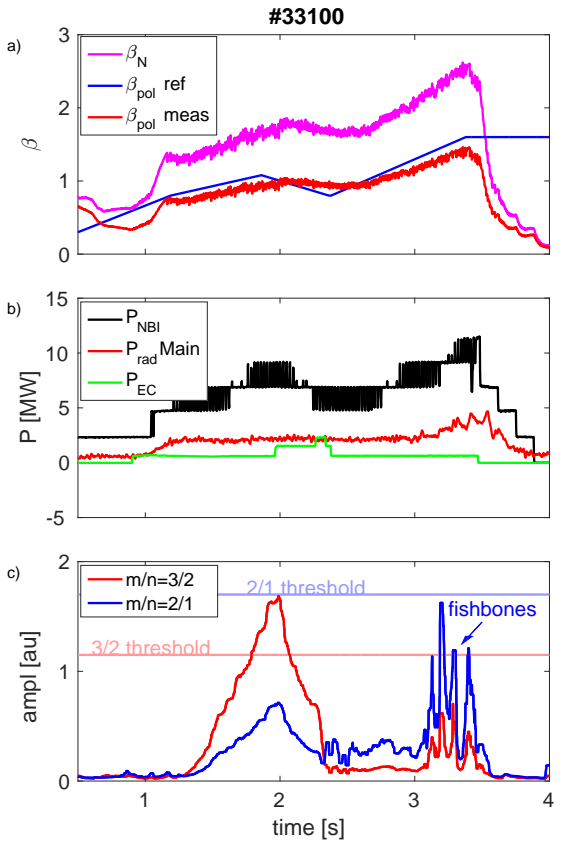

Figure 2: NTM and $\beta$ controllers working together. Frame (a) shows the plasma pressure, where $\beta_{p o l}$ is controlled to track a reference trajectory. $\beta_{N}$ is included for comparison. Frame (b) shows the input power from NBI and ECRH, compared to the power radiated in the main chamber. Frame (c) shows the amplitude of $3 / 2$ and 2/1 NTMs as measured by Mirnov coils.

A coupling was observed between NTMs and $T_{\text {div }}$, where NTMs in their growth phase increase transport and expel energy into the divertor. If the $T_{d i v}$ controller is aggressive and puffs a large amount of $N_{2}$, then main chamber radiation increases, $\beta$ is lowered and the NTM shrinks. Over time, the $N_{2}$ dissipates, $\beta$ increases and the cycle starts over. This should be taken into account when designing a multi-input multi-output (MIMO) controller.

$T_{d i v}$ and n0div are expected to be related. However, observations did not match the expectations. Usually, the quantities are inversely proportional to each other, for a constant power flux through the divertor. In frames (j-k) of fig. 3, both are well controlled close to their setpoints with oscillations which are decreasing in amplitude. Curiously, the phase between these oscillations is almost 0 , not 180 . No explanation has yet been found for this observation.

Control of the core density was hampered by fringe jumps and technical problems with the pellet launcher. Nevertheless, from $\mathrm{t}=1.8 \mathrm{~s}$ to $\mathrm{t}=3.0 \mathrm{~s}$ in frame (f) of fig. 3 we see the density raised to the set point by pellets being injected at the frequency shown in frame (e). Due to the transient density increase at the arrival of each 

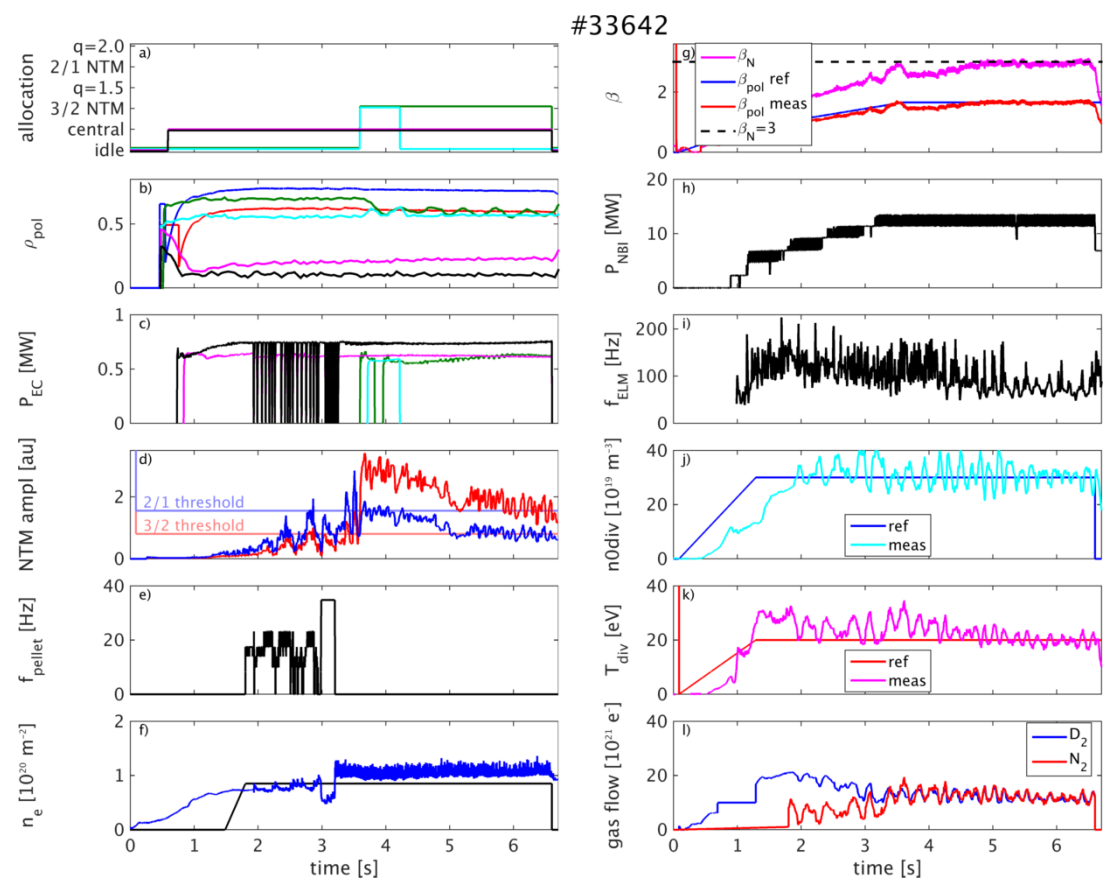

Figure 3: Excellent performance achieved using 5 SISO controllers. Frames (a-c) are as for fig. 1. Frame (d) shows the amplitude of 3/2 and 2/1 NTMs as measured by Mirnov coils. Frame (e) shows the pellet injection frequency, used to control the density. Measured density is shown in blue in frame(f), along with the reference in black. Frame(g) shows the plasma pressure as for fig. 2. Frame (h) shows the NBI power. Frame (i) shows the ELM frequency. Frames (j-k) show the neutral pressure and divertor temperature control, with reference and measurement trajectories. These controllers use the flow of $D_{2}$ and $N_{2}$ gas as actuators, shown in frame(l).

pellet, EC would be in cutoff and power would be reflected onto sensitive diagnostics. Therefore, as a precaution, EC is switched off briefly at the arrival of each pellet, giving the notched trace in frame (c). Obviously, the reduced duty cycle would affect the efficacy of EC for central heating and NTM stabilisation, however in this case there is enough margin that impurity accumulation does not become an issue and the estimate of the density after $\mathrm{t}=3.2 \mathrm{~s}$ is (erroneously) above the set point, such that the pellet frequency is set to $0 \mathrm{~Hz}$ before the NTM appears. The transient density increase to above the cutoff density may be specific to a device of AUG's size and/or dependent on the relative launch location of the pellets and ECRH. Further investigation is required.

Overall, the combination of all these controllers gave a very pleasing result, exemplified by \#33642 in fig. 3 . With 2 gyrotrons allocated to central heating throughout the discharge, the radiated power is kept low. When an NTM arises at $\mathrm{t}=3.6 \mathrm{~s}, 2$ gyrotrons are allocated to stabilise it. One of them trips at $t=4.2 \mathrm{~s}$, but the remaining gyrotron still manages to reduce the amplitude significantly, even while $\beta$ climbs back to the level it was at before the NTM - at constant pulse-width-modulated NBI power. $\beta_{N}$ is held stable above 3 for a relatively long time. The goal for the next campaign will be to demonstrate that feedback control enables reliable reproduction of this success. Improvements in fringe jump correction [8], the addition of main chamber radiation control using medium-Z impurities [9] and ELM control [10], as well as the introduction of integrated controllers may improve the performance even further.

\section{Conclusions}

An actuator management routine for ECRH was shown to perform exactly as designed, automatically selecting the optimal allocation of gyrotrons to targets in real-time. Configuration allows the algorithm to be tailored to a physicist's needs, and in the near future even to other devices such as TCV.

Control of NTMs and $\beta$ was integrated in a simple yet effective way, such that discharges reliably reached high $\beta$ values. In total, 5 feedback controllers were operated simultaneously. Couplings were observed between NTMs and $T_{d i v}, n 0 d i v$ and $T_{d i v}$, as well as pellets and EC. These will be taken into account in future designs of integrated controllers. 


\section{Acknowledgements}

This work has been carried out within the framework of the EUROfusion Consortium and has received funding from the Euratom research and training programme 2014-2018 under grant agreement No 633053. The views and opinions expressed herein do not necessarily reflect those of the European Commission.

[1] S. Qin, T. A. Badgwell, A survey of industrial model predictive control technology, Control Engineering Practice 11 (2003) 733 -764 .

[2] S. Skogestad, I. Postlethwaite, Multivariable Feedback Control: Analysis and Design, Wiley, 2005. URL: https://books . google.de/books?id=I6lsQgAACAAJ.

[3] I. Postlethwaite, et al., Industrial applications of advanced multivariable control, in: International Conference On Control 91, Vols 1 And 2, volume 332 of IEE CONFERENCE PUBLICATIONS, INST ELECT ENGINEERS, DIV COMP \& CONTROL; BRIT COMP SOC; CYBERNET SOC; IEEE, UKRO SECT; INST MATH \& ITS APPLICAT; INST MEASUREMENT \& CONTROL; INST PHYS; INST CHEM ENGINEERS; INST MECH ENGINEERS; INST NUCL ENGINEERS, 1991, pp. 729-733. 1991 INTERNATIONAL CONF ON CONTROL ( CONTROL 91 ), EDINBURGH, SCOTLAND, MAR 25-28, 1991.

[4] D. Moreau, et al., Integrated magnetic and kinetic control of advanced tokamak plasmas on DIII-D based on data-driven models, Nucl. Fusion 53 (2013) 063020.

[5] C. J. Rapson, et al., Actuator management for ECRH at ASDEX Upgrade, Fusion Eng. Des. 96 - 97 (2015) 694 - 697. Proceedings of the 28th Symposium On Fusion Technology (SOFT-28).

[6] E. Maljaars, Ongoing work on actuator management, Private Communication, 2016.

[7] P. T. Lang, et al., Controlled fuelling of high density scenarios at ASDEX Upgrade in support of ITER and DEMO, in: Europhysics Conference Abstracts, Proceedings of the 42nd EPS Plasma Physics Conference, Lisbon (2015) Paper 1.127, 2015, p. P1.127. URL: http://ocs.ciemat.es/ EPS2015ABS/pdf/P1.27.pdf.

[8] T. Blanken, et al., Particle density modeling for density profile reconstruction and fringe jump detection on TCV and ASDEX Upgrade, in: 43rd European Physical Society Conference on Plasma Physics (EPS), Leuven, Belgium, 4th July 2016., P4.031, 2016. URL: http: //ocs . ciemat.es/EPS2016PAP/ $\mathrm{pdf} / \mathrm{P} 4.031 . \mathrm{pdf}$.

[9] A. Kallenbach, et al., Optimized tokamak power exhaust with double radiative feedback in ASDEX Upgrade, Nucl. Fusion 52 (2012) 122003.

[10] M. Lennholm, et al., ELM frequency feedback control on JET, Nucl. Fusion 55 (2015) 063004. 